Article

\title{
Assessment of Bifidobacterium Species Using groEL Gene on the Basis of Illumina MiSeq High-Throughput Sequencing
}

\author{
Lujun Hu 1,2,+, Wenwei Lu 1,2,+ , Linlin Wang 1,2, Mingluo Pan 1,2, Hao Zhang 1,2,3, \\ Jianxin Zhao ${ }^{1,2,3, *}$ and Wei Chen $1,2,3,4$ \\ 1 State Key Laboratory of Food Science and Technology, Jiangnan University, Wuxi 214122, China; \\ 7130112038@vip.jiangnan.edu.cn (L.H.); luwenwei@jiangnan.edu.cn (W.L.); wanglllynn09@163.com (L.W.); \\ mingluopan@163.com (M.P.); zhanghao@jiangnan.edu.cn (H.Z.); chenwei66@jiangnan.edu.cn (W.C.) \\ 2 School of Food Science and Technology, Jiangnan University, Wuxi 214122, China \\ 3 National Engineering Research Center for Functional Food, Jiangnan University, Wuxi 214122, China \\ 4 Beijing Innovation Centre of Food Nutrition and Human Health, Beijing Technology and Business University, \\ Beijing 100048, China \\ * Correspondence: jxzhao@jiangnan.edu.cn; Tel.: +86-510-8591-2155 \\ + These authors contributed equally to this work.
}

Received: 31 October 2017; Accepted: 15 November 2017; Published: 21 November 2017

\begin{abstract}
The next-generation high-throughput sequencing techniques have introduced a new way to assess the gut's microbial diversity on the basis of $16 \mathrm{~S}$ rRNA gene-based microbiota analysis. However, the precise appraisal of the biodiversity of Bifidobacterium species within the gut remains a challenging task because of the limited resolving power of the $16 \mathrm{~S}$ rRNA gene in different species. The groEL gene, a protein-coding gene, evolves quickly and thus is useful for differentiating bifidobacteria. Here, we designed a Bifidobacterium-specific primer pair which targets a hypervariable sequence region within the groEL gene that is suitable for precise taxonomic identification and detection of all recognized species of the genus Bifidobacterium so far. The results showed that the novel designed primer set can specifically differentiate Bifidobacterium species from non-bifidobacteria, and as low as $10^{4}$ cells of Bifidobacterium species can be detected using the novel designed primer set on the basis of Illumina Miseq high-throughput sequencing. We also developed a novel protocol to assess the diversity of Bifidobacterium species in both human and rat feces through high-throughput sequencing technologies using groEL gene as a discriminative marker.
\end{abstract}

Keywords: Bifidobacterium; groEL; species level; biodiversity; MiSeq high-throughput sequencing

\section{Introduction}

Members of Bifidobacterium species, characterized by high G + C Gram-positive, non-motile, non-gas-producing, non-sporulating, anaerobic bacteria, constitute a group of the commensal bacterium of human and animal intestinal microbiota. The contribution of bifidobacteria in maintaining or improving human and animal health has been accepted and some members of the Bifidobacterium species have been added as probiotics to various foods [1,2]. Therefore, it is vital to detect and identify bifidobacterial strains precisely and to assess their diversity and population size in the gastrointestinal tract. Within this context, assessment of the Bifidobacterium species in complex samples such as feces from humans and animals has attracted great interest from researchers.

With the advent of massive parallel sequencing technologies, the cultivation-independent methods based on the 16S rRNA gene with the help of next-generation sequencing technologies have been extensively recognized as a useful tool for the assessment of Bifidobacterium species [3-7]. Among the nextgeneration sequencing technologies, the MiSeq Illumina sequencing platform had the lowest error rates 
and highest throughput per run as compared to the 454 GS Junior and Ion Torrent PGM [8]. However, the resolvability of the 16S rRNA gene sequences among closely related bacterial strains is limited. In general, bacterial strains showing more than 97\% $16 \mathrm{~S}$ rRNA gene sequence similarity are usually considered the same species. Because Bifidobacterium species reveal a relatively high $16 \mathrm{~S}$ rRNA gene sequence similarity, markers with higher discriminating power are necessary. The internal transcribed spacer (ITS) region provides a high resolution and can be used to assess population biodiversity in bacterial communities [9]. However, the ITS marker for studying the environmental samples is problematic in that the presence of operon copy number heterogeneity within a genome and the possibility of intragenomic variation in ITS sequence and length may lead to skewed estimates of bacterial communities $[10,11]$.

Alternative target genes, such as atpD [12], recA [13], tuf [14], dnaK [15], tal [16], xfp [17], rpoC [18], and groEL [19-22] have been used for the differentiation and identification of Bifidobacterium species. These gene markers have been demonstrated to have similar or even higher resolution ability for Bifidobacterium species than the $16 \mathrm{~S}$ rRNA gene. Compared to other molecular markers, the groEL gene has more advantages. The groEL gene is a ubiquitous housekeeping gene in the genus Bifidobacterium, that encodes the heat shock proteins (Hsp60, also known as Cpn60 or GroEL), which play an important role in response to cellular stress. Additional sequences of bifidobacterial strains are available in the Chaperonin Sequence Database [23]. Many experiments have proved that Bifidobacterium species have just one copy of the groEL gene [24-26], which facilitates quantitative analyses of Bifidobacterium species. Furthermore, the groEL gene sequence identities between Bifidobacterium species were much lower than those of $16 \mathrm{~S} \mathrm{rDNA}$, thus possessing higher resolution power for Bifidobacterium species than the 16S rRNA gene.

In this study, a novel protocol was described to assess Bifidobacterium species through high-throughput sequencing technologies using groEL as a discriminative marker. To test the robustness of the novel designed primer set, we analyzed Bifidobacterium species in human and rat fecal samples using the designed primer set on the basis of the MiSeq Illumina sequencing platform.

\section{Materials and Methods}

\subsection{Bacterial Strains, Culture Media and DNA Extraction}

The bifidobacterial strains used in the study were as follows: B. adolescentis CCFM626, B. animalis subsp. animalis CCFM624, B. animalis subsp. lactis BB12, B. bifidum CCFM641, B. breve CCFM623, B. dentium FJSNT63M4, B. longum subsp. infantis CCFM666, B. longum subsp. longum CCFM642, B. pseudocatenulatum CCFM749, and B. pseudolongum FJSWX2M9. All the bifidobacterial strains were grown in de Man, Rogosa and Sharp (MRS) broth with addition of $0.05 \%$ of L-cysteine hydrochloride monohydrate at $37^{\circ} \mathrm{C}$. Seven non-bifidobacterial strains were also used in this study: Actinomyces odontolyticus HNSQ3B4, Bacteroides uniformis CCFM792, Escherichia coli CCFM21, Enterococcus faecalis CCFM596, Lactobacillus acidophilus CCFM137, L. plantarum ST-III (CGMCC no. 0847) and Rothia dentocariosa JSWX1B7. All the Lactobacillus strains were cultured in MRS broth at $37^{\circ} \mathrm{C}$. E. coli was cultured at $37^{\circ} \mathrm{C}$ in Luria-Bertani medium. A. odontolyticus, B. uniformis, E. faecalis and R. dentocariosa were grown in Brain Heart Infusion Broth at $37^{\circ} \mathrm{C}$. All the bacteria used in this study came from the Culture Collection of Food Microorganisms of Jiangnan University (Wuxi, China).

Genomic DNA of these bacteria was extracted using the method described previously and subjected to further phenol/chloroform purification using an established protocol $[27,28]$.

\subsection{Fecal Sample Collection and Genomic DNA Extraction}

Feces from humans and from rats were collected in this study. All stool samples from adult humans were collected in sterile containers within $20 \mathrm{~min}$ after defecation, transported to the laboratory on ice, and stored at $-80{ }^{\circ} \mathrm{C}$ until genomic DNA was extracted. Fresh feces from rats were collected in individual sterile EP tubes on ice, which were taken to the laboratory within $2 \mathrm{~h}$ and stored at $-80^{\circ} \mathrm{C}$ for further observation. Fecal samples were homogenized and then subjected to bacterial genome 
DNA extraction using FastDNA SPIN Kit for Feces (MP Biomedicals; Carlsbad, CA, USA) as per the manufacturer's protocols. The protocols of the study were approved by the Ethical Committee of Jiangnan University (Wuxi, China).

\subsection{Phylogenetic Analysis}

The phylogenetic relationships among the genus Bifidobacterium were constructed using groEL gene. The best fitted substitution model for each partition was estimated using Akaike information criterion (AIC) implemented in jModeltest [29]. The model of TIM1 + I + G was chosen for Maximum likelihood (ML) analyses, which were performed with RAxML BlackBox web servers [30].

\subsection{Bifidobacterium groEL-Specific Primer Design}

All available bifidobacterial and some actinobacterial groEL gene sequences were retrieved from the GenBank and European Molecular Biology Laboratory (EMBL) nucleotide sequence databases and aligned using the ClustalW software program [31]. To identify Bifidobacterium species, a region of 487 or 496 base pairs (bp) located at positions 1066 to 1552 (B. animalis) or 1561 (B. breve) of the complete groEL gene of ca. $1600 \mathrm{bp}$ was chosen as discriminative target sites. Degenerate primers Bif-groEL-F (5-TCC GAT TAC GAY CGY GAG AAG CT-3)/Bif-groEL-R (5-CSG CYT CGG TSG TCA GGA ACA G-3) for the genus Bifidobacterium were manually designed according to multiple sequence alignment. Specificity testing was carried out using PRIMER-BLAST which allows in silico PCR amplification using the National Center for Biotechnology Information (NCBI) nonredundant database as a template [32]. An additional specificity test was conducted by PCR amplification using genomic DNA extracted from known bacterial species: A. odontolyticus, B. uniformis, E. coli, E. faecalis, L. acidophilus, L. plantarum and $R$. dentocariosa. The primers were synthesized by the Shanghai Sangon Biological Science \& Technology Company (Shanghai, China) and used for the PCR amplification.

\subsection{PCR Amplification, Quantification, and Sequencing}

The selected partial groEL gene sequences from microbial genome DNA were PCR amplified using the barcoded fusion primers Bif-groEL-F/Bif-groEL-R designed during this study. PCR amplifications were performed using a $50 \mu \mathrm{L}$ total volume consisting of $1 \mu \mathrm{L}$ of the target DNA, $25 \mu \mathrm{L}$ Premix Taq (TaKaRa, Dalian, China), $1 \mu \mathrm{L}$ of each primer $(20 \mu \mathrm{M})$ and $22 \mu \mathrm{L}$ of double distilled water $\left(\mathrm{ddH}_{2} \mathrm{O}\right)$. The PCR amplification procedures were pre-denaturation at $95{ }^{\circ} \mathrm{C}$ for $4 \mathrm{~min}$, followed by 30 cycles consisting of denaturation at $95{ }^{\circ} \mathrm{C}$ for $30 \mathrm{~s}$, annealing for $30 \mathrm{~s}$ at $60^{\circ} \mathrm{C}$, extension for $50 \mathrm{~s}$ at $72{ }^{\circ} \mathrm{C}$ and the final step at $72{ }^{\circ} \mathrm{C}$ for $10 \mathrm{~min}$. In addition, The V3-V4 hypervariable sequence region of the $16 \mathrm{~S}$ rRNA gene was amplified with the forward primer (341F: 5-CCT AYG GGR BGC ASC AG-3) and reverse primer (806R: 5-GGA CTA CNN GGG TAT CTA AT-3) according to the previous protocols [33].

All the PCR amplification products obtained following amplification of the groEL and 16S rRNA gene sequences were excised from the agarose gel, purified using a QIAquick Gel Extraction Kit (Qiagen, Hilden, Germany) and quantified by Quant-iT PicoGreen dsDNA Assay Kit (Life Technologies, Carlsbad, CA, USA) following the manufacturer's recommendations. DNA amplicon libraries were prepared using TruSeq DNA LT Sample Preparation Kit (Illumina, San Diego, CA, USA) and sequenced on the MiSeq Illumina platform using the MiSeq v3 Reagent Kit (600 cycles) following instructions provided by the manufacturer.

\subsection{Evaluation of the Sensitivity of the Novel Designed Primer Set}

The detection sensitivity and accuracy of the primer set Bif-groEL-F/Bif-groEL-R were evaluated employing known DNA amounts, ranging from 0.01 to $40 \mathrm{ng}$, of the artificial sample from 10 different bifidobacterial taxa. The groEL gene copy numbers were estimated using "Calculator for Determining the Number of Copies of a Template" (URI Genomics \& Sequencing Center) [34]. Thus, colony-forming units (CFU) could be calculated on the basis of the groEL gene copy numbers predicted above. 


\subsection{Bioinformatic Sequence Analysis}

Sequence reads were processed with the QIIME package version 1.9.1 (Quantitative Insights Into Microbial Ecology, Flagstaff, AZ, USA) [35]. The raw sequences with a lower quality score and short-read length compared to target sequences were first removed. Sequences were also removed if they contained ambiguous bases or mismatches in primers. Only pair-end reads overlapping longer than $10 \mathrm{bp}$ and without any mismatch were assembled following their overlap sequences, and the unassembled reads were discarded. Barcode and sequencing primers from the above assembled sequences were trimmed.

Each sample's high-quality reads were clustered into operational taxonomic units (OTUs) for further taxonomic analyses. Taxonomic identification of OTUs for the groEL sequences was performed through comparison to the Chaperonin Sequence Database [23]. The OTUs of V3-V4 region sequences were assigned to a taxonomy with the naive Bayes classifier of the Ribosomal Database Project (RDP) [36], and all OTUs with representative sequences from each cluster were combined and aligned against the Greengenes core set in QIIME with the PyNAST aligner [35,37]. Similarities among the microbial communities were estimated using cluster heatmap analysis with the R software for statistical computing [38].

\subsection{Real-Time $q P C R$}

The total and main bifidobacterial numbers of human feces were determined by SYBR Green-based qPCR using a CFX96 real-time PCR detection system (Bio-Rad Laboratories, Hercules, CA, USA) as previously described with some modifications [22,39]. The total bifidobacteria and the main bifidobacteria of B. pseudocatenulatum and B. longum subsp. longum from human feces were quantified through qPCR amplifications using the primers previously described in $20 \mu \mathrm{L}$ volume using 96-well plates in triplicate [22]. For quantification of the genus Bifidobacterium, B. longum subsp. longum CCFM642 was used as the standard strain. B. pseudocatenulatum CCFM749 and B. longum subsp. longum CCFM642 were also used as qPCR controls for species-specific quantification. The target bifidobacterial population was expressed as $\log _{10}$ bifidobacteria per gram of wet stool.

\subsection{Statistical Analysis}

All data were expressed as means \pm standard deviation (SD). The statistical analyses of differences between two groups were analyzed using Student's $t$-test. The analyses were carried out with SPSS version 16.0 (SPSS Inc., Chicago, IL, USA), and statistical significance was accepted at least at the $5 \%$ level.

\section{Results}

\subsection{Comparative Analysis of the groEL and $16 S \mathrm{rRNA}$ Gene}

The groEL and 16S rRNA gene nucleotide sequences of Bifidobacterium species (download from NCBI and EMBL databases) were compared by BLAST [40] (Table 1). When comparing the two sets of sequences, we found that the lowest value of the pairwise similarities of the groEL gene is $79.1 \%$ (B. magnum and B. tsurumiense) and the average pairwise similarities value is $86.3 \%$ whereas the lowest value of the pairwise similarities of the 16S rRNA gene is $90.9 \%$ (B. magnum and B. crudilactis) and the average pairwise similarities value is $93.8 \%$. Thus, the groEL gene provids higher resolution power than that provided by the $16 \mathrm{~S}$ rRNA gene. We also plotted the average pairwise similarities values targeted for the groEL gene against the average pairwise similarities values corresponding to $16 \mathrm{~S}$ rRNA gene sequences for classification of microorganisms at the same taxonomic rank. Using the regression shown in Figure 1, we found that the variation trend of the average pairwise similarities values were similar between the groEL and 16S rRNA genes. 
Table 1. List of Bifidobacterium strains used for comparative analysis.

\begin{tabular}{|c|c|c|c|c|}
\hline Number & Bifidobacterium Species & Strain ${ }^{a}$ & GenBank Accession no. of groEL Gene Sequences & GenBank Accession no. of $16 \mathrm{~S}$ rRNA Gene Sequences \\
\hline $\begin{array}{l}1 \\
2\end{array}$ & $\begin{array}{l}\text { B. adolescentis } \\
\text { B. angulatum }\end{array}$ & $\begin{array}{c}\text { ATCC } 15703 \\
\text { JCM } 7096\end{array}$ & $\begin{array}{l}\text { AP009256 } \\
\text { AP012322 }\end{array}$ & $\begin{array}{c}\text { NR_074802 } \\
\text { LC071846 }\end{array}$ \\
\hline 3 & B. animalis subsp. animalis & ATCC $25527=$ LMG 10508 & СР002567 & JGYM01000004 \\
\hline 4 & B. animalis subsp. lactis & BB12 & СР001853 & GU116483 \\
\hline 5 & B. asteroides & Bin2 & NZ_KQ033859 & EF187231 \\
\hline 6 & B. biavatii & DSM 23969 & JGYN01000004 & JGYN01000007 \\
\hline 7 & B. bifidum & PRL2010 & СР001840 & СР001840 \\
\hline 8 & B. bohemicum & R53250 & FMAM01000001 & FMAM01000014 \\
\hline 9 & B. boum & LMG $10736=$ JCM 1211 & JGYQ01000016 & LC071799 \\
\hline 10 & B. breve & JCM 1192 & AP012324 & LC071793 \\
\hline 11 & B. callitrichos & DSM 23973 & JGYS01000001 & JGYS01000004 \\
\hline 12 & B. catenulatum & DSM 16992 & AP012325 & NR_041875 \\
\hline 13 & B. choerinum & ATCC $27686=$ LMG 10510 & JGYU01000001 & D86186 \\
\hline 14 & B. coryneforme & Bma6 & KQ033865 & EF187237 \\
\hline 15 & B. crudilactis & LMG 23609 & NZ_JHAL01000002 & NZ_JHAL01000001 \\
\hline 16 & $\begin{array}{l}\text { B. crutuntects } \\
\text { B. cuniculi }\end{array}$ & LMG 10738 & JGYV01000008 & JX986964 \\
\hline 17 & B. dentium & JCM 1195 & AP012326 & LCO71795 \\
\hline 18 & B. gallicum & DSM 20093 = LMG 11596 & NZ_ABXB03000002 & ABXB03000004 \\
\hline 19 & B. gallinarum & DSM 20670 = JCM 6291 & NZ_JDUN01000004 & D86191 \\
\hline 20 & B. indicum & LMG $11587=$ DSM $20214=$ JCM1302 & СР006018 & D86188 \\
\hline 21 & B. magnum & DSM $20222=$ JCM 1218 & NZ_ATVE01000001 & D86193 \\
\hline 22 & B. merycicum & DSM $6492=$ JCM 8219 & NZ_JDTL01000006 & D86192 \\
\hline 23 & B. minimum & DSM $20102=$ ATCC 27538 & NZ_ATXM01000001 & M58741 \\
\hline 24 & B. kashiwanohense & HM2-1 & AB578933 & AB491757 \\
\hline 25 & B. longum subsp. infantis & ATCC 15697 & CP001095 & NR_043437 \\
\hline 26 & B. longum subsp. longum & BBMN68 & СР002286 & GQ380695.1 \\
\hline 27 & B. mongoliense & DSM 21395 & JGZE01000001 & AB433856 \\
\hline 28 & B. pseudocatenulatum & JCM 1200 & AP012330 & $\begin{array}{l}\text { ADD50000 } \\
\text { LC071796 }\end{array}$ \\
\hline 29 & B. pseudolongum & PV8-2 & СР007457 & СР007457 \\
\hline 30 & B. pullorum & DSM 20433 = JCM 1214 & NZ_JDUI01000001 & D86196 \\
\hline 31 & B. reuteri & DSM 23975 & NZ_JDUW01000002 & NZ_JDUW01000049 \\
\hline 32 & B. ruminantium & DSM $6489=$ JCM 8222 & NZ_JHWQ01000003 & D86197 \\
\hline 33 & B. saeculare & DSM 6531 & JGZM01000001 & D89328 \\
\hline 34 & B. scardovii & JCM 12489 & AP012331 & AP012331 \\
\hline 35 & B. stellenboschense & DSM 23968 & NZ_JGZP01000019 & JGZP01000012 \\
\hline 36 & B. stercoris & JCM 15918 & JGZQ01000008 & NZJJDUX01000017 \\
\hline 37 & B. subtile & DSM 20096 & NZ_AUFH01000005 & D89378 \\
\hline 38 & B. thermacidophilum subsp. porcinum & LMG 21689 & JGZS01000003 & NZJJGZS01000003 \\
\hline 39 & B. thermophilum & RBL67 & СР004346 & DQ340557 \\
\hline 40 & B. tsurumiense & DSM $17777=$ OMB115 & NZ_AUCL01000007 & AB241106 \\
\hline
\end{tabular}

a ATCC, American Type Culture Collection; DSM, Deutsche Sammlung von Mikroorganismen und Zellkulturen; JCM, Japanese Collection of Microorganisms; LMG, Laboratorium voor Microbiologie, University of Ghent. 


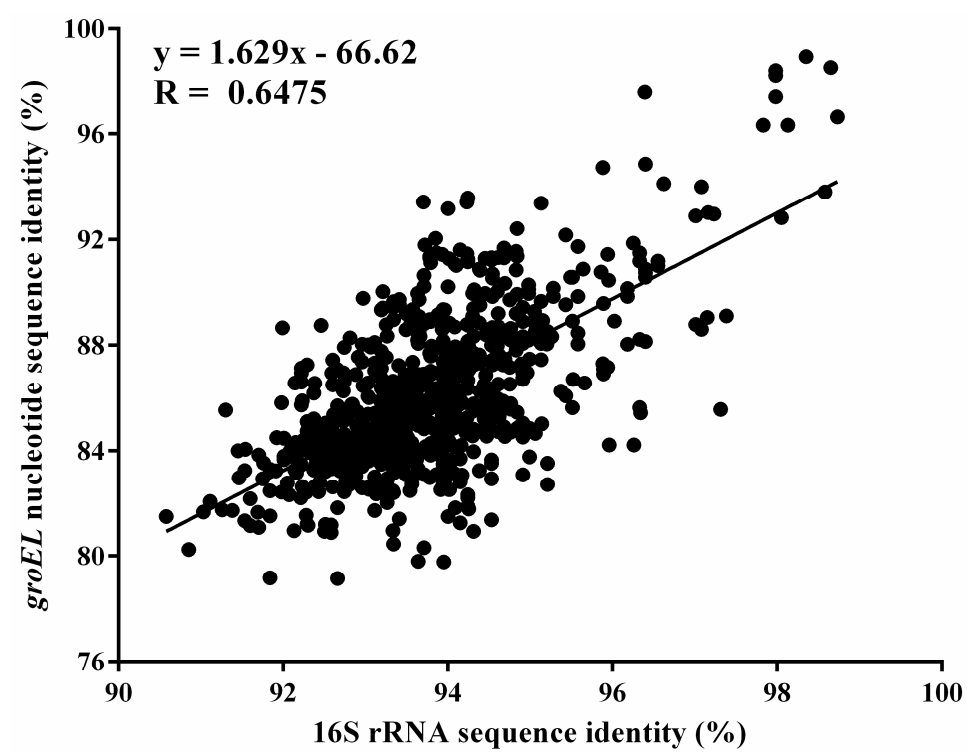

Figure 1. Association between the degree of sequence identity of $16 \mathrm{~S}$ rDNA and the groEL gene for pairs of genomes assigned to the same species.

\subsection{Phylogenetic Analysis of the Partial groEL Gene}

The study determined the selected partial sequences of the groEL gene and V3-V4 region of the 16S rRNA gene of 120 bifidobacterial strains from 54 species (subspecies) (Table S1). The results by BLAST revealed that the lowest value of the pairwise similarities of the partial groEL gene is $74.9 \%$ (B. commune R-52791 and B. animalis subsp. lactis BB12) and the average pairwise similarities value is $84.9 \%$. We used the MEGA software (Version 5.1) to align the selected partial groEL gene and V3-V4 region sequences determined in this study [41]. As shown in Figure 2, a ML analysis of groEL gene sequences from the bifidobacterial strains conducted in the phylogenetic dendrogram revealed that the Bifidobacterium species were grouped into six clusters. Moreover, the closely related Bifidobacterium species (i.e., subspecies) fell into the same clusters, whereas different Bifidobacterium species were categorized into different clusters. Specifically, the closely related taxa such as members of $B$. longum subsp. longum, B. longum subsp. infantis and B. longum subsp. suis, as well as B. animalis subsp. animalis and $B$. animalis subsp. lactis can be distinguished on the basis of the selected partial groEL gene, confirming that the partial groEL gene possessed the high taxonomic and phylogenetic resolution for identification and differentiation of the Bifidobacterium species. However, as shown in Figure S1, the neighbor-joining tree on the basis of the V3-V4 region sequences of the 16S rRNA gene showed that some of the same Bifidobacterium species were grouped into different clusters, demonstrating that the V3-V4 region sequences of the 16S rRNA gene lacked sufficient resolution for distinguishing different Bifidobacterium species. 


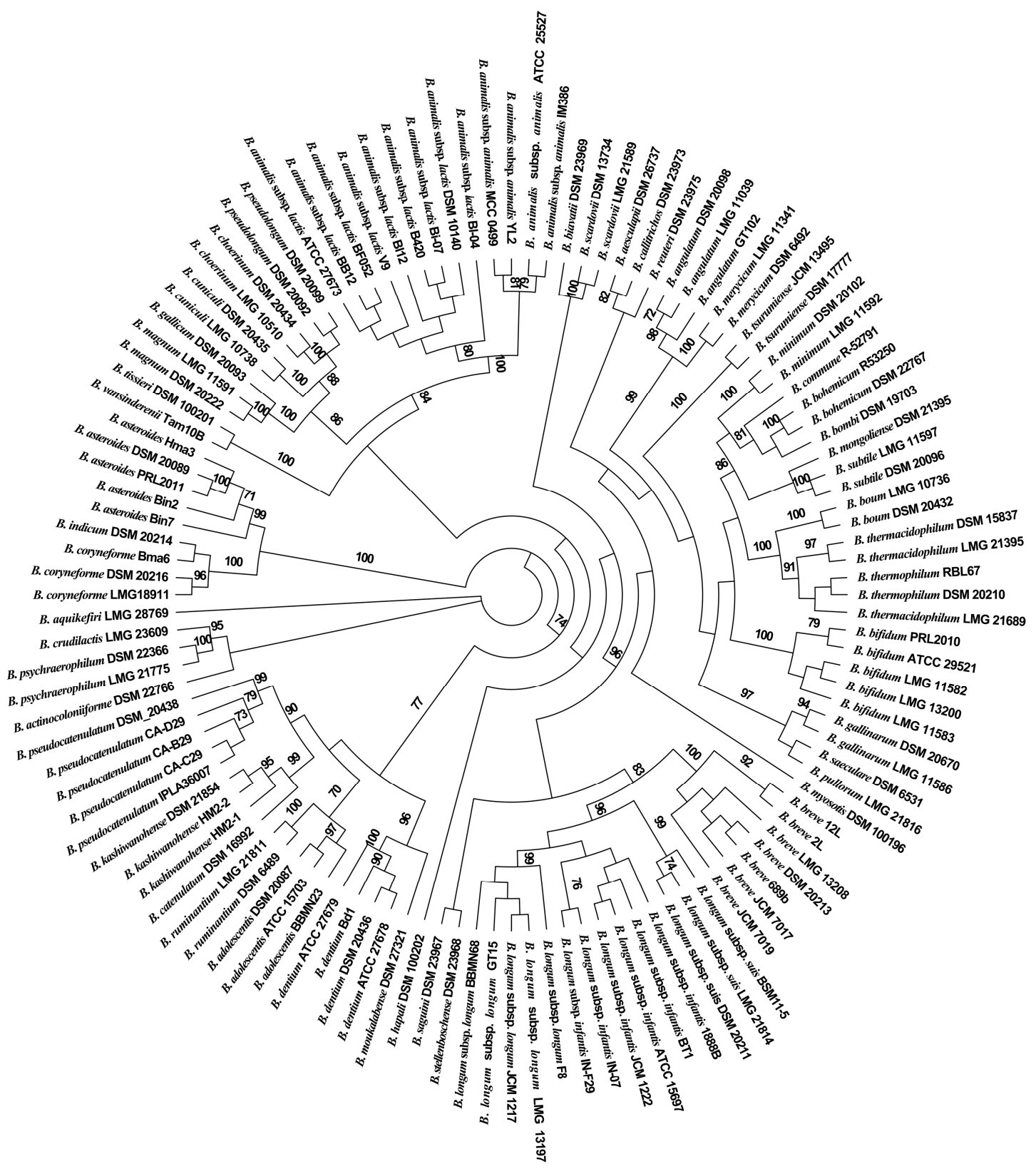

Figure 2. A maximum likelihood phylogeny of the selected partial groEL gene sequences for the genus Bifidobacterium. Bootstrap values above $70 \%$ are given on the branches based on 1000 replicates of the phylogenetic tree.

\subsection{Specificity, Accuracy and Sensitivity of the Novel Designed Primer Set}

In silico PCR through PRIMER-BLAST generates only an amplicon for bifidobacterial genomes, suggesting the bifidobacterial specificity of the primer set. We also performed PCR amplification using genomic DNA extracted from known bacterial species including ten bifidobacterial strains and seven non-bifidobacterial strains. As shown in Figure 3, the results revealed that a PCR amplification product was obtained only when template DNA was extracted from Bifidobacterium species, whereas no PCR amplification product was observed when DNA genome from any of the other investigated non-bifidobacterial strains was used as a template. Therefore, the novel designed primer set can specifically differentiate Bifidobacterium species from other bacterial species tested in this study. 


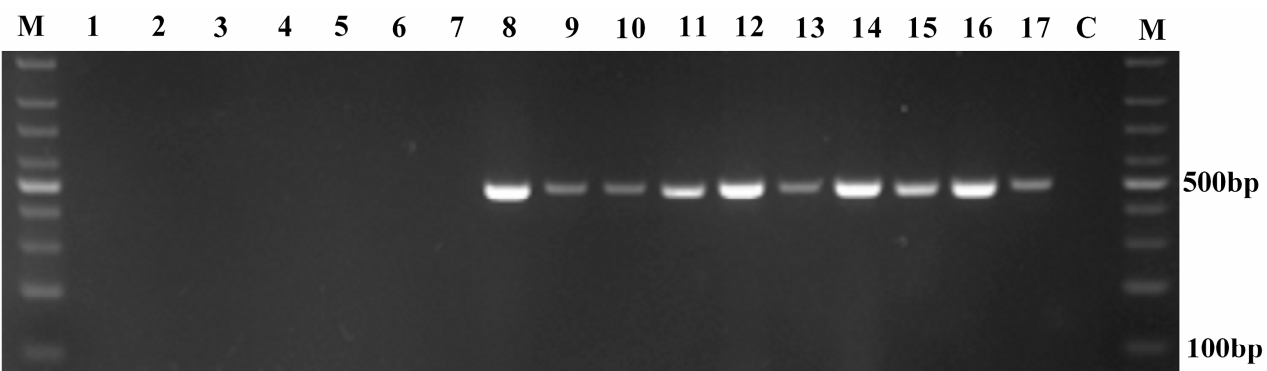

Figure 3. Specificity of PCR amplification of the selected partial groEL gene using the novel designed primer set. M, marker; 1, A. odontolyticus, 2, B. uniformis, 3, E. coli, 4, E. faecalis, 5, L. acidophilus, 6, L. plantarum; 7, R. dentocariosa, 8, B. adolescentis; 9, B. animalis subsp. animalis; 10 , B. animalis subsp. lactis; 11 , B. bifidum; 12 , B. breve; 13 , B. dentium; 14 , B. longum subsp. infantis; 15 , B. longum subsp. longum; 16 , B. pseudocatenulatum; 17, B. pseudolongum; C, control.

To evaluate the accuracy and sensitivity of the novel designed primer set, we developed artificial samples consisting of known DNA amounts of different bifidobacterial species. The genomic DNA from these Bifidobacterium species served as a template for PCR amplification with the novel designed primer set, and the amplicons were sequenced on the MiSeq Illumina sequencing platform. Figure 4A shows strong correlation of the relative abundances of taxa through comparison between known bifidobacterial composition of the artificial samples and retrieved results through groEL-profiling analysis. Specifically, the minimum DNA amount of detectable bifidobacterial species was $0.05 \mathrm{ng}$, which corresponds to Bifidobacterium species of $10^{4} \mathrm{CFU}$ at concentration. Therefore, the limit of detection (LOD) of the novel designed primer set based on the MiSeq Illumina sequencing platform was $10^{4} \mathrm{CFU} / \mathrm{mL}$ (Figure $4 \mathrm{~B}$ ).

A

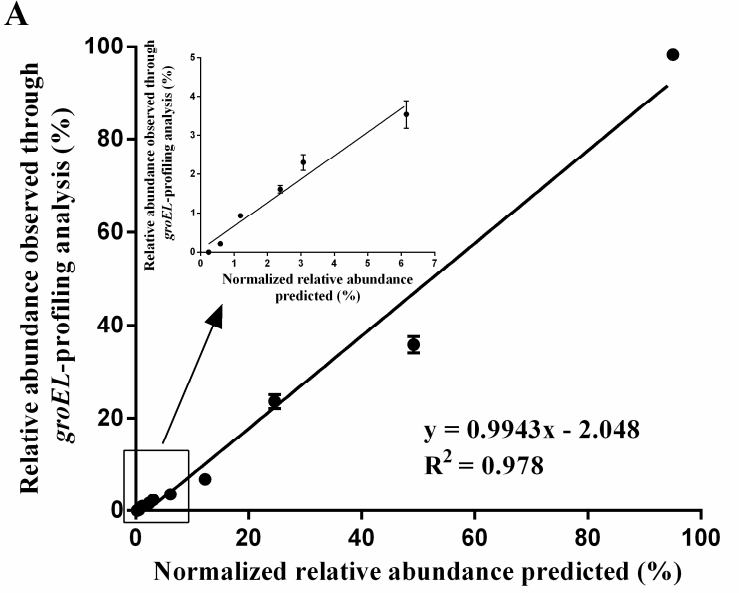

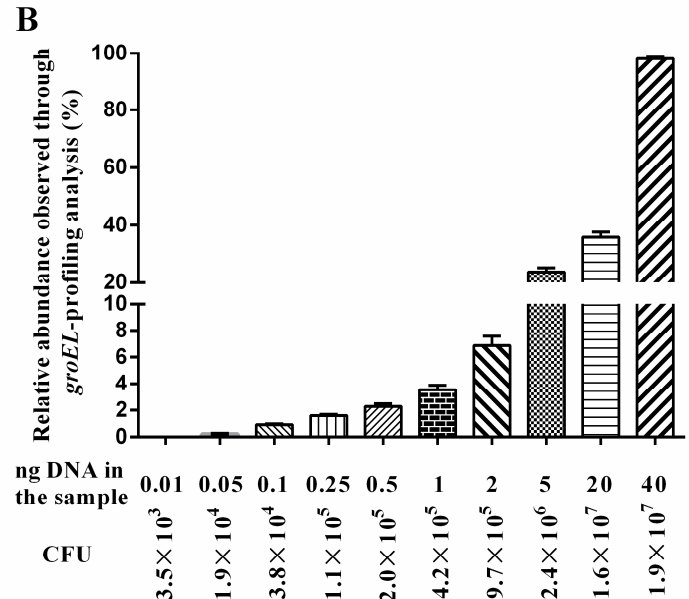

Figure 4. Accuracy and the limit of detection of the novel designed primer set. (A) Relationship between normalized relative abundance predicted of Bifidobacterium species and relative abundance observed through groEL-profiling analysis. (B) The limit of detection (LOD) of the novel designed primer set based on the selected partial groEL gene. CFU: Colony-forming units.

\subsection{Comparison of Resolving Power between the Partial groEL Gene and V3-V4 Region of $16 S$ rRNA}

To evaluate the efficacy of the groEL-based primer set designed in the study, we sequenced the partial groEL gene and V3-V4 region of 16S rRNA amplicons obtained by PCR amplification using the same genomic DNA from human and rat fecal samples. As shown in Table 2, MiSeq sequencing analysis of feces samples from eight humans generated 136,488 and 181,257 high-quality and classifiable sequences corresponding to the groEL gene and $16 \mathrm{~S}$ rRNA gene, respectively, and average sequence reads of 
the two genes were 17,061 and 22,657 per sample. For eight rat fecal samples, 150,875 and 152,033 high-quality and classifiable sequences were obtained for the groEL gene and $16 \mathrm{~S}$ rRNA gene, respectively, and average sequence reads of the two genes were 18,859 and 19,004 per sample.

To evaluate the robustness of the designed primer pair in determining the bifidobacterial community composition in complex ecosystems, the bifidobacterial profiles identified were compared using the new designed primer pair Bif-groEL-F/Bif-groEL-R and the bifidobacterial profiles obtained with the primer set $341 \mathrm{~F} / 806 \mathrm{R}$ as described previously in each case using the same genomic DNA. As shown in Figure $5 \mathrm{~A}, \mathrm{~B}$, when assessing the diversity of bifidobacteria, using the universal primer set corresponding to the $\mathrm{V} 3-\mathrm{V} 4$ region of the $16 \mathrm{~S}$ rRNA gene, on average, only about $2.8 \%$ and $14.4 \%$ of the tens of thousands of reads generated for the human and rat fecal samples were assigned to the genus Bifidobacterium. When using the primer pairs Bif-groEL-F/Bif-groEL-R, we amplified a region of the groEL gene from Bifidobacterium species, and the results revealed that almost all the sequences could be assigned to the genus Bifidobacterium (Figure 5C,D). Furthermore, the primer pairs targeted to the partial groEL gene could identify bifidobacteria at the species level, in contrast to the universal primer set of the V3-V4 region of $16 \mathrm{~S}$ rDNA at the genus level.

A

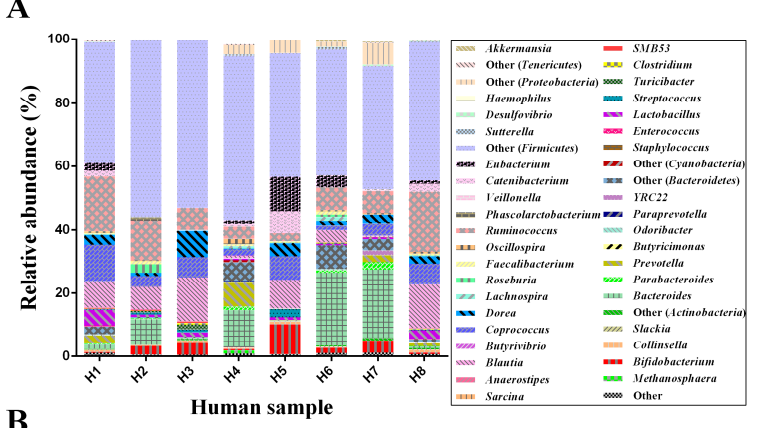

B

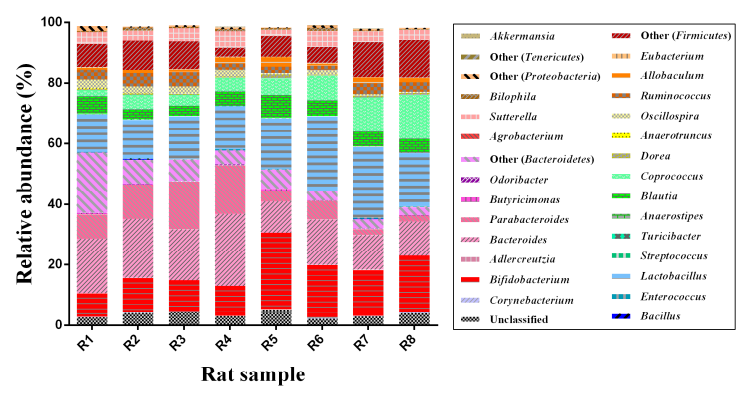

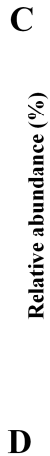

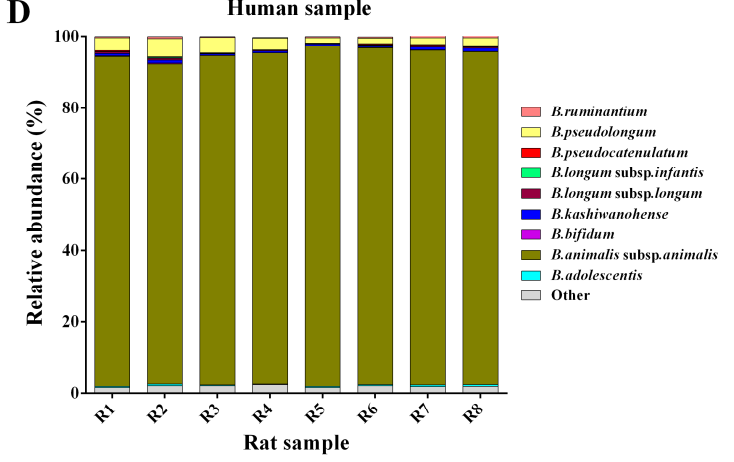

Figure 5. 16S rRNA gene-based and groEL gene-based profiling of human and rat fecal samples involving Bif-groEL-F/Bif-groEL-R and 341F/806R primer pairs. (A) Bar plots of the microbial composition at the genus level of the eight analyzed human samples and (B) of the eight analyzed rat samples. (C) Bar plots of the microbial composition at the species level of the eight analyzed human samples and (D) of the eight analyzed rat samples.

Table 2. Overview of sequencing results for each sample.

\begin{tabular}{|c|c|c|c|c|}
\hline Sample ID & Sequence Number ${ }^{\text {a }}$ (16S) & OTU Number ${ }^{b}(16 S)$ & Sequence Number (groEL) & OTU Number (groEL) \\
\hline $\mathrm{H} 2$ & 29297 & 1566 & 20519 & 3050 \\
\hline $\mathrm{H} 4$ & 28524 & 2632 & 18206 & 3176 \\
\hline H5 & 18197 & 970 & 15605 & 2536 \\
\hline H6 & 19625 & 2045 & 15297 & 3943 \\
\hline
\end{tabular}


Table 2. Cont.

\begin{tabular}{ccccc}
\hline Sample ID & Sequence Number $^{\mathbf{a}} \mathbf{( 1 6 S )}$ & OTU Number $^{\mathbf{b}} \mathbf{( 1 6 S )}$ & Sequence Number $\left(\right.$ groEL) $^{\text {OTU Number }(\mathbf{g r o E L})}$ \\
\hline R1 & 13986 & 1351 & 9341 & 736 \\
R2 & 9721 & 1279 & 9385 & 815 \\
R3 & 13187 & 1462 & 34297 & 1665 \\
R4 & 16230 & 1404 & 27903 & 1545 \\
R5 & 43429 & 936 & 17599 & 967 \\
R6 & 19969 & 596 & 19835 & 1060 \\
R7 & 18249 & 723 & 13146 & 1111 \\
R8 & 17262 & 930 & 870 \\
\hline
\end{tabular}

${ }^{a}$ The sequence number refers to the count of assembled sequences after quality filtering. ${ }^{b}$ The OTU (Operational Taxonomic Units) number is presented for all sequences without rarefaction.

\subsection{Comparison of Bifidobacterium Species between Humans and Rats}

As shown in Figure 5C,D, 12 Bifidobacterium species are contained in human fecal samples, and nine are contained in rat fecal samples. Notably, the predominant Bifidobacterium species for human gut bacteria were B. pseudocatenulatum and B. longum subsp. longum. The dominate gut bifidobacteria of rats were $B$. animalis subsp. animalis. Furthermore, when cluster heatmap analysis (Figure 6) was performed to visualize the differences in the composition of Bifidobacterium species from human and rat fecal samples, these Bifidobacterium species from humans and rats formed two distinct blocks on the heatmap. Notably, the relative abundances of B. pseudocatenulatum and B. longum subsp. longum (humans) and $B$. animalis subsp. animalis (rats) are significantly different among gut bifidobacteria from humans and rats $(p<0.05)$ (Figure 7).

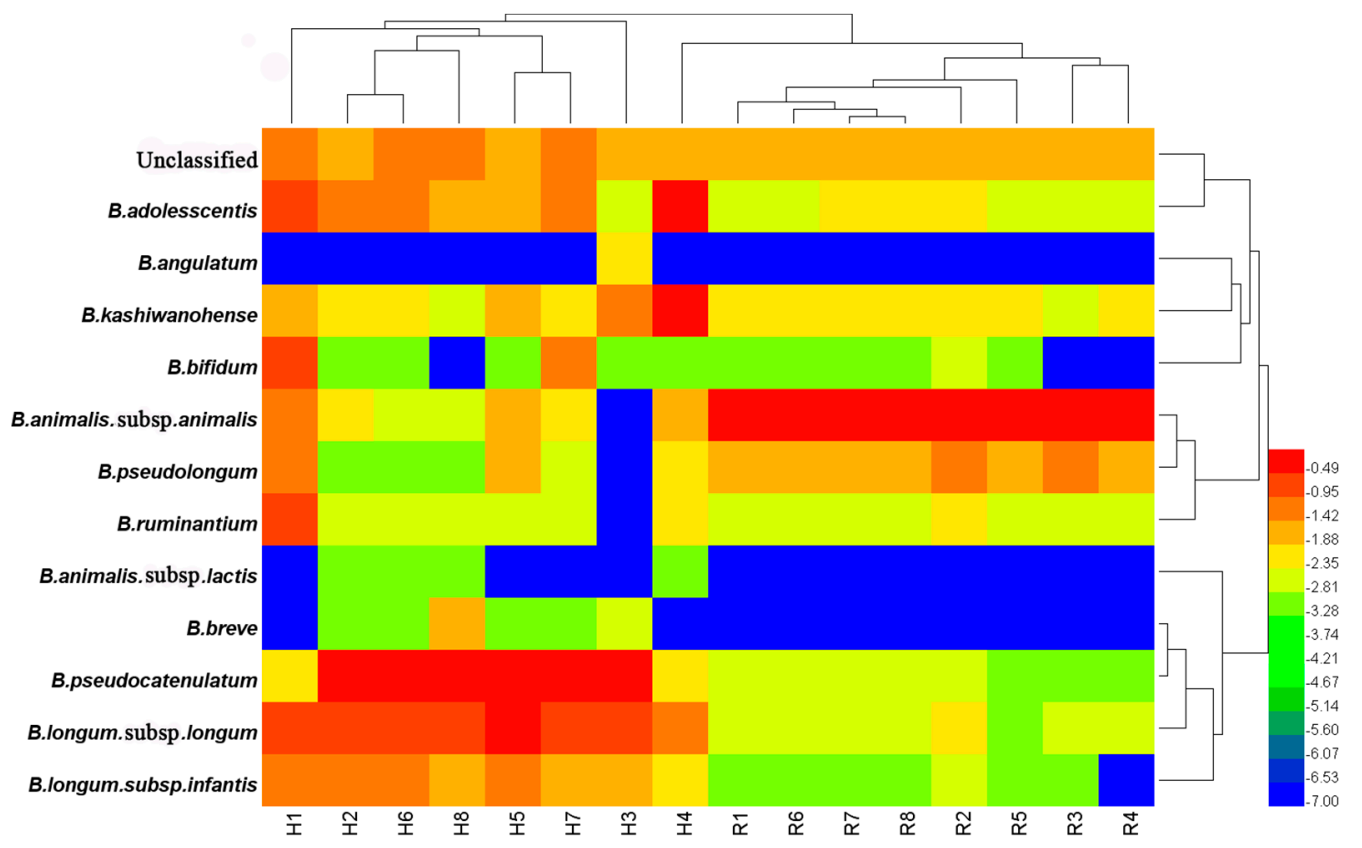

Figure 6. Relative abundance of Bifidobacterium species was visualized using a heatmap determined using the sequence data obtained from human and rat fecal samples, with a high percentage of species belonging to the genus Bifidobacterium indicated in red and low percentages in blue. Each row on the $y$-axis represents a Bifidobacterium species, and each column on the $x$-axis represents a sample. 


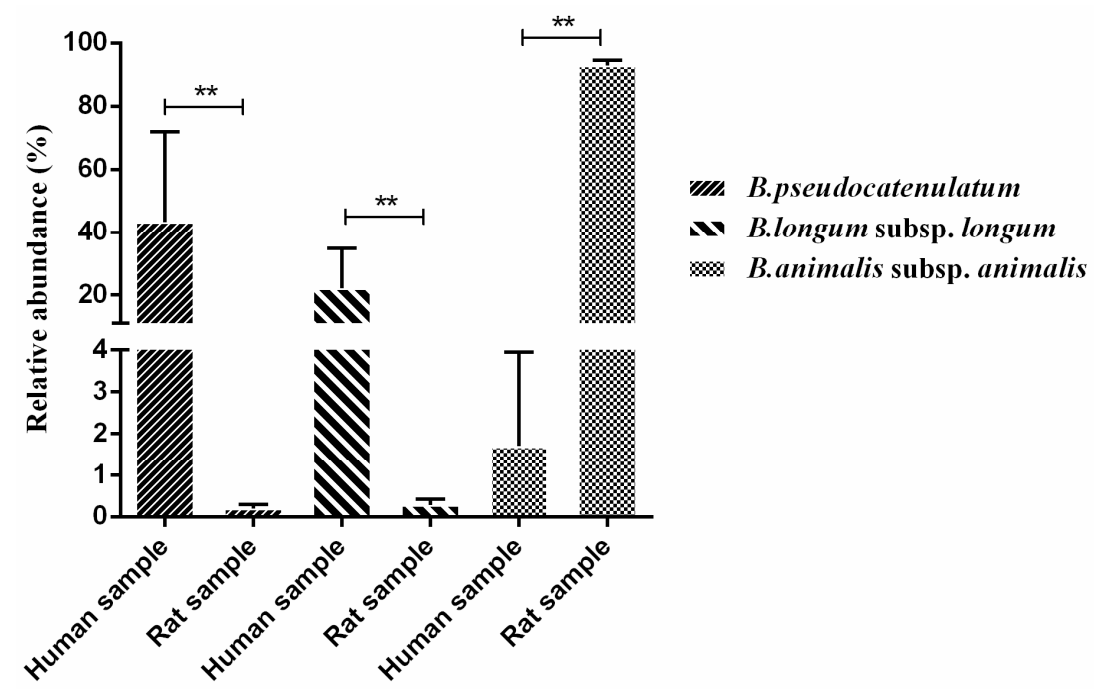

Figure 7. Differences in relative abundance of selected Bifidobacterium species. ${ }^{*} p<0.05 ;{ }^{* *} p<0.01$ according to one-way analysis of variance and Duncan's multiple comparisons test.

\section{6. qPCR-Based Determination of Main Bifidobacterial Numbers}

To further quantify the main bifidobacterial numbers in human feces, qPCR analysis was carried out in a CFX96 real-time PCR detection system. As shown in Table S2, the results of the main bifidobacterial community composition in human feces from qPCR were in accord with those from the new method of using the designed primer set on the basis of the MiSeq Illumina sequencing platform.

\section{Discussion}

To identify Bifidobacterium species on the basis of the MiSeq Illumina sequencing platform, the following criteria should be met: (1) The target gene must be ubiquitous in the genus Bifidobacterium; (2) the target gene must have high resolution power; (3) the target gene used for primer binding must include a sequence containing a hypervariable region flanked by two constant regions; (4) the PCR amplification region in the target gene must comprise no more than 500-bp nucleotide sequences; and (5) many sequences of the target gene must be available.

The groEL gene, a single-copy housekeeping gene, is ubiquitous in the genus Bifidobacterium. According to the multiple sequence alignment method used in the study, we selected a fragment of about $490 \mathrm{bp}$ for PCR amplification with the designed degenerate primers, and the selected partial groEL nucleotide sequence identities among different Bifidobacterium species ranged from 74.3 to $96.7 \%$ (mean $85.0 \%$ ). In addition, the phylogenetic tree depicted using the selected region of the groEL gene or even complete groEL gene nucleotide sequences (about $1600 \mathrm{bp}$ ) of Bifidobacterium species [18,20,21] was similar to the one based on the 16S rRNA gene sequences [3,42]. A significant correlation existed between the genetic distances of the groEL gene nucleotide sequences and those of the $16 \mathrm{~S}$ rDNA nucleotide sequences [21]. Furthermore, the $16 \mathrm{~S}$ rDNA nucleotide sequence identities among all the Bifidobacterium species ranged from 90.6 to $99.9 \%$ [42]. Notably, the resolving ability of the $16 \mathrm{~S}$ rRNA gene is limited among some closely related Bifidobacterium species. For example, B. catenulatum and B. pseudocatenulatum cannot be distinguished because they share $98.5 \%$ nucleotide sequence similarity in their 16S rRNA genes sequences [18]. However, the phylogenetic dendrogram delineated in this study showed that B. catenulatum and B. pseudocatenulatum could be easily distinguished because of the $93.9 \%$ nucleotide sequence similarity between the two organisms in the selected region of the groEL gene. Moreover, at the subspecies level, B. animalis subsp. animalis and B. animalis subsp. lactis, as well as B. longum subsp. infantis, B. longum subsp. longum and B. longum subsp. suis, share $16 \mathrm{~S}$ rRNA nucleotide sequence similarities are all above $99 \%$ [43]. The selected region of the groEL gene nucleotide 
sequence identities between B. animalis subsp. animalis and B. animalis subsp. lactis, as well as B. longum subsp. infantis and B. longum subsp. longum were $94.1 \%$ and $98.2 \%$, respectively. In contrast, the selected region of the groEL gene used during the study displayed a considerably higher resolving ability between these closely related species in Bifidobacterium species than did the 16S rRNA gene. What is more, the reference databases (NCBI, EMBL and Chaperonin Sequence Database) provide ample nucleotide sequences of the groEL gene for further sequence alignment. Overall, the selected region of the groEL gene fulfils all of the prerequisites to serve as a reliable alternative target marker gene for distinguishing different Bifidobacterium species based on the MiSeq Illumina sequencing platform.

To characterize the specificity and LOD of the developed method using the novel designed primer set on the high-throughput sequencing platform, we prepared samples spiked with the known concentrations of Bifidobacterium species and other bacteria for sequencing based on the MiSeq Illumina platform. The results demonstrated that the developed method could discriminate different Bifidobacterium species, and concentrations of Bifidobacterium species as low as $10^{4} \mathrm{CFU} / \mathrm{mL}$ could be detected.

To prove the robustness of the novel designed primer set, two kinds of different fecal samples derived from humans and rats were selected for further analysis. Based on the MiSeq Illumina sequencing platform, we identified the Bifidobacterium species of both sets of fecal samples. The results showed that the diversity of bifidobacterial composition in human samples is greater than that in rat samples. One possible reason may be that diets varied among different people, whereas diets in different rats did not vary.

It is known that there may be drawbacks to each method. Maybe there is no one gene that can differentiate all Bifidobacterium species. One possible limitation of groEL gene is that a high similarity of groEL gene similarity values between different bifidobacterial species is present. For example, there are high similarity values between B. thermophilum and B. thermacidophilum subsp. thermacidophilum, B. longum subsp. infantis and B. longum subsp. longum. Consequently, we should be careful when differentiating these different Bifidobacterium species using groEL gene. Maybe groEL gene combined with more genes like $16 \mathrm{~S}$ rRNA, $r p o B$ and $\operatorname{cl} p C$ are good in differentiating these different bifidobacterial species with a high similarity of groEL similarity values [44].

\section{Conclusions}

In conclusion, a Bifidobacterium-specific primer pair that targets a hypervariable region of about $490 \mathrm{bp}$ within the groEL gene can specifically differentiate Bifidobacterium species from bacterial species, and the LOD of the novel designed primer set based on the MiSeq Illumina sequencing platform was $10^{4} \mathrm{CFU} / \mathrm{mL}$. In addition, the novel designed primer pair can identify bifidobacteria at the species level from human and rat fecal samples on the basis of the MiSeq Illumina sequencing platform. The results demonstrated that the predominant Bifidobacterium species for human gut bacteria were B. pseudocatenulatum and B. longum subsp. longum, and the predominant gut bifidobacteria of rats were B. animalis subsp. animalis.

Supplementary Materials: The following are available online at www.mdpi.com/2073-4425/8/11/336/s1. Figure S1: Phylogenetic tree based on the V3-V4 region sequences of the 16S rRNA gene. The tree was constructed by the neighbor-joining distance method with bootstrap values calculated from 1000 trees, Table S1: List of Bifidobacterium strains used for phylogenetic analysis of the selected partial groEL gene and the V3-V4 region of the $16 \mathrm{~S}$ rRNA gene.

Acknowledgments: The work was supported by the Key Program of the National Natural Science Foundation of China (Grant No. 31530056).

Author Contributions: Lujun $\mathrm{Hu}$, Wenwei $\mathrm{Lu}$ and Jianxin Zhao conceived and designed the experiments; Lujun Hu and Wenwei Lu performed the experiments; Lujun Hu and Jianxin Zhao analyzed the data; Linlin Wang, Mingluo Pan, Hao Zhang and Wei Chen contributed reagents/materials/analysis tools; and Lujun Hu and Jianxin Zhao wrote the paper.

Conflicts of Interest: The authors declare no conflict of interest. 


\section{References}

1. Arvanitoyannis, I.S.; Van Houwelingen-Koukaliaroglou, M. Functional foods: A survey of health claims, pros and cons, and current legislation. Crit. Rev. Food Sci. Nutr. 2005, 45, 385-404. [CrossRef] [PubMed]

2. Backhed, F.; Ley, R.E.; Sonnenburg, J.L.; Peterson, D.A.; Gordon, J.I. Host-bacterial mutualism in the human intestine. Science 2005, 307, 1915-1920. [CrossRef] [PubMed]

3. Turroni, F.; Marchesi, J.R.; Foroni, E.; Gueimonde, M.; Shanahan, F.; Margolles, A.; van Sinderen, D.; Ventura, M. Microbiomic analysis of the bifidobacterial population in the human distal gut. ISME J. 2009, 3, 745-751. [CrossRef] [PubMed]

4. Fouhy, F.; Guinane, C.M.; Hussey, S.; Wall, R.; Ryan, C.A.; Dempsey, E.M.; Murphy, B.; Ross, R.P.; Fitzgerald, G.F.; Stanton, C.; et al. High-throughput sequencing reveals the incomplete, short-term recovery of infant gut microbiota following parenteral antibiotic treatment with ampicillin and gentamicin. Antimicrob. Agents Chemother. 2012, 56, 5811-5820. [CrossRef] [PubMed]

5. Claesson, M.J.; Jeffery, I.B.; Conde, S.; Power, S.E.; O'Connor, E.M.; Cusack, S.; Harris, H.M.B.; Coakley, M.; Lakshminarayanan, B.; O'Sullivan, O.; et al. Gut microbiota composition correlates with diet and health in the elderly. Nature 2012, 488, 178-184. [CrossRef] [PubMed]

6. Milani, C.; Hevia, A.; Foroni, E.; Duranti, S.; Turroni, F.; Lugli, G.A.; Sanchez, B.; Martín, R.; Gueimonde, M.; van Sinderen, D.; et al. Assessing the fecal microbiota: An optimized ion torrent 16S rRNA gene-based analysis protocol. PLoS ONE 2013, 8, e68739. [CrossRef] [PubMed]

7. Mao, B.; Li, D.; Ai, C.; Zhao, J.; Zhang, H.; Chen, W. Lactulose differently modulates the composition of luminal and mucosal microbiota in C57BL/6J mice. J. Agric. Food Chem. 2016, 64, 6240-6247. [CrossRef] [PubMed]

8. Loman, N.J.; Misra, R.V.; Dallman, T.J.; Constantinidou, C.; Gharbia, S.E.; Wain, J.; Pallen, M.J. Performance comparison of benchtop high-throughput sequencing platforms. Nat. Biotechnol. 2012, 30, 434-439. [CrossRef] [PubMed]

9. Milani, C.; Lugli, G.A.; Turroni, F.; Mancabelli, L.; Duranti, S.; Viappiani, A.; Mangifesta, M.; Segata, N.; van Sinderen, D.; Ventura, M. Evaluation of bifidobacterial community composition in the human gut by means of a targeted amplicon sequencing (ITS) protocol. FEMS Microbiol. Ecol. 2014, 90, 493-503. [CrossRef] [PubMed]

10. Stewart, F.J.; Cavanaugh, C.M. Intragenomic variation and evolution of the internal transcribed spacer of the rRNA operon in bacteria. J. Mol. Evol. 2007, 65, 44-67. [CrossRef] [PubMed]

11. Crosby, L.D.; Criddle, C.S. Understanding bias in microbial community analysis techniques due to RRN operon copy number heterogeneity. Biotechniques 2003, 34, 790-802. [PubMed]

12. Ventura, M.; Canchaya, C.; van Sinderen, D.; Fitzgerald, G.F.; Zink, R. Bifidobacterium lactis DSM 10140: Identification of the atp (atpBEFHAGDC) operon and analysis of its genetic structure, characteristics, and phylogeny. Appl. Environ. Microbiol. 2004, 70, 3110-3121. [CrossRef] [PubMed]

13. Ventura, M.; Zink, R. Comparative sequence analysis of the $t u f$ and $r e c A$ genes and restriction fragment length polymorphism of the internal transcribed spacer sequences supply additional tools for discriminating Bifidobacterium lactis from Bifidobacterium animalis. Appl. Environ. Microbiol. 2003, 69, 7517-7522. [CrossRef] [PubMed]

14. Ventura, M.; Canchaya, C.; Meylan, V.; Klaenhammer, T.R.; Zink, R. Analysis, characterization, and loci of the tuf genes in Lactobacillus and Bifidobacterium species and their direct application for species identification. Appl. Environ. Microbiol. 2003, 69, 6908-6922. [CrossRef] [PubMed]

15. Ventura, M.; Zink, R.; Fitzgerald, G.F.; van Sinderen, D. Gene structure and transcriptional organization of the dnaK operon of Bifidobacterium breve UCC 2003 and application of the operon in bifidobacterial tracing. Appl. Environ. Microbiol. 2005, 71, 487-500. [CrossRef] [PubMed]

16. Requena, T.; Burton, J.; Matsuki, T.; Munro, K.; Simon, M.A.; Tanaka, R.; Watanabe, K.; Tannock, G.W. Identification, detection, and enumeration of human Bifidobacterium species by PCR targeting the transaldolase gene. Appl. Environ. Microbiol. 2002, 68, 2420-2427. [CrossRef] [PubMed]

17. Yin, X.; Chambers, J.R.; Barlow, K.; Park, A.S.; Wheatcroft, R. The gene encoding xylulose-5-phosphate/fructose-6phosphate phosphoketolase $(x f p)$ is conserved among Bifidobacterium species within a more variable region of the genome and both are useful for strain identification. FEMS Microbiol. Lett. 2005, 246, 251-257. [CrossRef] [PubMed] 
18. Ventura, M.; Canchaya, C.; Del Casale, A.; Dellaglio, F.; Neviani, E.; Fitzgerald, G.F.; van Sinderen, D. Analysis of bifidobacterial evolution using a multilocus approach. Int. J. Syst. Evol. Microbiol. 2006, 30, 734-759. [CrossRef] [PubMed]

19. Zhu, L.; Li, W.; Dong, X. Species identification of genus Bifidobacterium based on partial HSP60 gene sequences and proposal of Bifidobacterium thermacidophilum subsp. porcinum subsp. nov. Int. J. Syst. Evol. Microbiol. 2003, 53, 1619-1623. [CrossRef] [PubMed]

20. Masco, L.; Ventura, M.; Zink, R.; Huys, G.; Swings, J. Polyphasic taxonomic analysis of Bifidobacterium animalis and Bifidobacterium lactis reveals relatedness at the subspecies level: Reclassification of Bifidobacterium animalis as Bifidobacterium animalis subsp. animalis subsp. nov. and Bifidobacterium lactis as Bifidobacterium animalis subsp. lactis subsp. nov. Int. J. Syst. Evol. Microbiol. 2004, 54, 1137-1143. [PubMed]

21. Ventura, M.; Canchaya, C.; Zink, R.; Fitzgerald, G.F.; van Sinderen, D. Characterization of the groEL and groES loci in Bifidobacterium breve UCC 2003: Genetic, transcriptional, and phylogenetic analyses. Appl. Environ. Microbiol. 2004, 70, 6197-6209. [CrossRef] [PubMed]

22. Junick, J.; Blaut, M. Quantification of human fecal Bifidobacterium species by use of quantitative real-time PCR analysis targeting the groEL gene. Appl. Environ. Microbiol. 2012, 78, 2613-2622. [CrossRef] [PubMed]

23. Hill, J.E.; Penny, S.L.; Crowell, K.G.; Goh, S.H.; Hemmingsen, S.M. cpnDB: A chaperonin sequence database. Genome Res. 2004, 14, 1669-1675. [CrossRef] [PubMed]

24. Schell, M.A.; Karmirantzou, M.; Snel, B.; Vilanova, D.; Berger, B.; Pessi, G.; Zwahlen, M.C.; Desiere, F.; Bork, P.; Delley, M.; et al. The genome sequence of Bifidobacterium longum reflects its adaptation to the human gastrointestinal tract. Proc. Natl. Acad. Sci. USA 2002, 99, 14422-14427. [CrossRef] [PubMed]

25. Garrigues, C.; Johansen, E.; Pedersen, M.B. Complete genome sequence of Bifidobacterium animalis subsp. lactis BB-12, a widely consumed probiotic strain. J. Bacteriol. 2010, 192, 2467-2468. [CrossRef] [PubMed]

26. Zhurina, D.; Zomer, A.; Gleinser, M.; Brancaccio, V.F.; Auchter, M.; Waidmann, M.S.; Westermann, C.; van Sinderen, D.; Riedel, C.U. Complete genome sequence of Bifidobacterium bifidum S17. J. Bacteriol. 2011, 193, 301-302. [CrossRef] [PubMed]

27. Ventura, M.; Reniero, R.; Zink, R. Specific identification and targeted characterization of Bifidobacterium lactis from different environmental isolates by a combined multiplex-PCR approach. Appl. Environ. Microbiol. 2001, 67, 2760-2765. [CrossRef] [PubMed]

28. Sambrook, J.; Russell, D.W. Molecular Cloning: A Laboratory Manual, 3rd ed.; Cold Spring Harbor Laboratory Press: New York, NY, USA, 2001.

29. Posada, D. jModelTest: Phylogenetic model averaging. Mol. Biol. Evol. 2008, 25, 1253-1256. [CrossRef] [PubMed]

30. Stamatakis, A.; Hoover, P.; Rougemont, J. A rapid bootstrap algorithm for the RAxML web servers. Syst. Biol. 2008, 57, 758-771. [CrossRef] [PubMed]

31. Chenna, R.; Sugawara, H.; Koike, T.; Lopez, R.; Gibson, T.J.; Higgins, D.G.; Thompson, J.D. Multiple sequence alignment with the Clustal series of programs. Nucleic Acids Res. 2003, 31, 3497-3500. [CrossRef] [PubMed]

32. Ye, J.; Coulouris, G.; Zaretskaya, I.; Cutcutache, I.; Rozen, S.; Madden, T.L. Primer-BLAST: A tool to design target-specific primers for polymerase chain reaction. BMC Bioinformatics 2012, 13, 134. [CrossRef] [PubMed]

33. Jia, H.R.; Geng, L.L.; Li, Y.H.; Wang, Q.; Diao, Q.Y.; Zhou, T.; Dai, P.L. The effects of Bt Cry1Ie toxin on bacterial diversity in the midgut of Apis mellifera ligustica (Hymenoptera: Apidae). Sci. Rep. 2016, 6, 24664. [CrossRef] [PubMed]

34. Staroscik, A. Calculator for Determining the Number of Copies of a Template. Available online: http:/ / cels.uri. edu/gsc/cndna.html (accessed on 8 February 2017).

35. Caporaso, J.G.; Kuczynski, J.; Stombaugh, J.; Bittinger, K.; Bushman, F.D.; Costello, E.K.; Fierer, N.; Peña, A.G.; Goodrich, J.K.; Gordon, J.I.; et al. QIIME allows analysis of high-throughput community sequencing data. Nat. Methods 2010, 7, 335-336. [CrossRef] [PubMed]

36. Wang, Q.; Garrity, G.M.; Tiedje, J.M.; Cole, J.R. Naive Bayesian classifier for rapid assignment of rRNA sequences into the new bacterial taxonomy. Appl. Environ. Microbiol. 2007, 73, 5261-5267. [CrossRef] [PubMed]

37. Caporaso, J.G.; Bittinger, K.; Bushman, F.D.; DeSantis, T.Z.; Andersen, G.L.; Knight, R. PyNAST: A flexible tool for aligning sequences to a template alignment. Bioinformatics 2010, 26, 266-267. [CrossRef] [PubMed]

38. R Development Core Team. R: A Language and Environment for Statistical Computing; R Foundation for Statistical Computing: Vienna, Austria, 2011. 
39. Lewisa, Z.T.; Bokulicha, N.A.; Kalanetraa, K.M.; RuizMoyanoa, S.; Underwoodb, M.A.; Mills, D.A. Use of bifidobacterial specific terminal restriction fragment length polymorphisms to complement next generation sequence profiling of infant gut communities. Anaerobe 2013, 19, 62-69. [CrossRef] [PubMed]

40. Altschul, S.F.; Madden, T.L.; Schäffer, A.A.; Zhang, J.; Zhang, Z.; Miller, W.; Lipman, D.J. Gapped BLAST and PSI-BLAST: A new generation of protein database search programs. Nucleic Acids Res. 1997, 25, 3389-3402. [CrossRef] [PubMed]

41. Tamura, K.; Peterson, D.; Peterson, N.; Stecher, G.; Nei, M.; Kumar, S. MEGA5: Molecular evolutionary genetics analysis using maximum likelihood, evolutionary distance, and maximum parsimony methods. Mol. Biol. Evol. 2011, 28, 2731-2739. [CrossRef] [PubMed]

42. Miyake, T.; Watanabe, K.; Watanabe, T.; Oyaizu, H. Phylogenetic analysis of the genus Bifidobacterium and related genera based on $16 \mathrm{~S}$ rDNA sequences. Microbiol. Immunol. 1998, 42, 661-667. [CrossRef] [PubMed]

43. Jian, W.; Zhu, L.; Dong, X. New approach to phylogenetic analysis of the genus Bifidobacterium based on partial HSP60 gene sequences. Int. J. Syst. Evol. Microbiol. 2001, 51, 1633-1638. [CrossRef] [PubMed]

44. Duranti, S.; Mangifesta, M.; Lugli, G.A.; Turroni, F.; Anzalone, R.; Milani, C.; Mancabelli, L.; Ossiprandi, M.C.; Ventura, M. Bifidobacterium vansinderenii sp. nov., isolated from faeces of emperor tamarin (Saguinus imperator). Int. J. Syst. Evol. Microbiol. 2017, 67, 3987-3995. [CrossRef] [PubMed]

(C) 2017 by the authors. Licensee MDPI, Basel, Switzerland. This article is an open access article distributed under the terms and conditions of the Creative Commons Attribution (CC BY) license (http:/ / creativecommons.org/licenses/by/4.0/). 